EPJ Web of Conferences 29, 00005 (2012)

DOI: $10.1051 /$ epjconf/20122900005

C) Owned by the authors, published by EDP Sciences, 2012

\title{
Influence of the materials magnetic state on the accurate determination of the magnetocaloric effect
}

\author{
M. Balli ${ }^{\mathrm{a} 1}$, O. Sari ${ }^{1,2}$, D. Fruchart ${ }^{3}$ and J. Forchelet ${ }^{1}$ \\ ${ }^{1}$ University of Applied Sciences of Western Switzerland, Institute of Thermal Sciences and \\ Engineering, CH-1401 Yverdon-les-Bains, Switzerland \\ ${ }^{2}$ Clean Cool Systems Company, C/O ADNV, place de la tannerie 1400 Yverdon-Les-Bains, \\ Switzerland \\ ${ }^{3}$ Néel Institute, département MCMF, CNRS, Avenue des Martyrs 25, Grenoble, France.
}

\begin{abstract}
In this paper, we report a detailed study of the magnetocaloric effect (MCE) in different first order magnetic transition (FOMT) materials with different situation of the magnetic state (magnetic order). For this purpose, $\mathrm{R}-\mathrm{Co}_{2}, \mathrm{MnAs}$ based compounds were considered in this study. The MCE is discussed in terms of Maxwell relation (MR) and Clausius-Clapeyron (C-C) equation. The deviation observed between both methods is discussed and analyzed. On the other hand, practically all the reported data of the MCE in the literature are associated to the applied external magnetic field and have not been corrected taking into account the demagnetization effect related to the materials shape. The obtained results demonstrate that this phenomenon can alter drastically the MCE values by cancelling out a large part of the external field, resulting in spurious values of the measured MCE. The effect of the demagnetization field on the magnetocaloric performances is also the subject of this paper.
\end{abstract}

\section{Introduction}

In recent years, magnetic refrigeration based on the magnetocaloric effect (MCE) has become one of the best candidates for new cooling technology instead of the gas-vaporization refrigeration. This is due to its potential for more efficient and environmentally friendly refrigeration technology. Magnetic refrigeration does not rely on hydrofluorocarbons (HCFs) used in conventional refrigeration systems. HCFs are powerful greenhouse gases that contribute to global climate change when they escape into atmosphere. The MCE, discovered by Warburg in 1881 [1], is defined as the temperature change of a material when it is subjected to a magnetic field. In particular, this intrinsic property is observed in magnetic materials which contain $3 \mathrm{~d}$ metals and lanthanide elements. Under the effect of the magnetic field, the magnetic order is changed and correspondingly the magnetic entropy part is increased or decreased, depending on the nature of the magnetocaloric materials: paramagnets, ferromagnets, anti-ferromagnets...etc. The adiabatic condition assures entropy conservation leading to the lattice entropy change, causing consequently, a variation in temperature of the magnetic material. The MCE is large if the field has a significant impact on the order parameter, i.e. at low temperatures in paramagnets and near the order-disorder transition temperature

a e-mail : mohamed.balli@heig-vd.ch

This is an Open Access article distributed under the terms of the Creative Commons Attribution License 2.0, which permits unrestricted use, distribution, and reproduction in any medium, provided the original work is properly cited. 
in ferromagnets and anti-ferromagnets. In addition of the magnetic field, the MCE depends strongly on numerous intrinsic and extrinsic factors. The crystalline structure, the chemical composition, the shape and the magnetic state of a material are among the most important parameters that define its MCE.

For applications close to room temperature, gadolinium metal $\left(\mathrm{T}_{\mathrm{C}}=294 \mathrm{~K}\right)$ was used early as refrigerant, but its cost, its poor resistance to oxidation and additionally its moderate value of MCE limit its applications. Consequently, materials with high MCE are needed for the development of magnetic refrigeration. For this purpose, various transition elements-based compounds and rare earth intermetallic alloys have so far been reported as potential refrigerant materials for different working temperature range [2-9]. Generally, most of materials are characterized by their isothermal entropy change $\Delta \mathrm{S}$, which is calculated using the Maxwell relation and experimentally determined from isothermal magnetization. However, the use of Maxwell relation to determine the entropy change related to MCE, in first order magnetic transition (FOMT) materials, has been the subject of controversy and many discussions [10-17]. In their paper, Giguère et al. [10] have shown that the entropy change calculated from the Maxwell relation was overestimated (at least by $20 \%$ ) compared to that obtained from the Clausius-Clapeyron $(\mathrm{C}-\mathrm{C})$ equation. They also claimed that the adiabatic temperature change calculated from heat capacity is much overestimated when compared to direct measurements. Therefore, they concluded that the Maxwell relation was not usable for materials with first order magnetic transition such as $\mathrm{Gd}_{5} \mathrm{Si}_{2} \mathrm{Ge}_{2}$. Instead, the Clausius-Clapeyron equation, that relates the magnetization jump at the transition and the shifts of the critical temperature with magnetic field, is more appropriated. In their response, Gshneidner et al [11], have confirmed that, the Maxwell relation can be applied even for a $1^{\text {st }}$ order transition, except for an ideal $1^{\text {st }}$ order phase transition occurring infinitely fast at constant T, P and H. Moreover, they claimed that the ClausiusClapeyron equation would imply a temperature step independent of the field step, which is not the case for the experimental data reported by Giguère et al [10]. In addition, more recently Balli et al [14] and Liu et al [15] demonstrated that the thermodynamic Maxwell's relation becomes invalid especially when a magnetic material maintained close to the transition, mixes paramagnetic and ferromagnetic phases. On the other hand, practically all the reported data of the MCE in the literature are associated to the applied external magnetic field and have not been corrected taking into account the demagnetization effect related to the materials shape. The demagnetization field can alter drastically the MCE values by cancelling out a large part of the external field, resulting in spurious values of the measured MCE.

For a correct evaluation of the MCE, in this paper we aim to contribute by analyzing MCE in different kind of materials with FOMT materials frequently studied and encountered in the literature. Depending on the nature of the magnetic order and the existing phases in the compounds, the MCE is discussed in terms of Maxwell relation and Clausius-Clapeyron (C-C) equation. The deviation observed between both methods for different kind of materials is discussed and analyzed. On the other hand, the effect of the demagnetization field on the correct determination of the MCE is also studied. Considering Gd as case of study, a methodology is proposed to evaluate accurately the MCE.

\section{First order materials with phase-separated state}

Nowadays, most research on magnetocaloric materials are focused on materials undergoing a first order magnetic phase transition because of their large MCE in particular the isothermal entropy change $\triangle \mathrm{S}$. The giant MCE reported in this kind of materials is attributed generally to an abrupt change of the magnetization associated with the structural transformation near the transition point. MnAs compound and its derivatives are good prototypes for magnetic refrigerants with FOMT. MnAs is a ferromagnet with saturation magnetization of $3.4 \mu_{\mathrm{B}} / \mathrm{Mn}$. A first-order ferromagnetic to paramagnetic transition takes place around $318 \mathrm{~K}$. This transition is accompanied by a structural transition from hexagonal NiAs type to an orthorhombic MnP-type structure. Wada et al [3] have reported a giant MCE in MnAs close to room temperature. Under a 0-5 T field variation, $-\Delta \mathrm{S}$ and 
$\Delta \mathrm{T}_{\mathrm{ad}}$ were found to be about $30 \mathrm{~J} / \mathrm{kg} \mathrm{K}$ and $13 \mathrm{~K}$, respectively. However, as with the cases of other materials known to undergo first-order transitions, the temperature-induced transition in the MnAs compound can be field-induced in the reverse direction by the application of a magnetic field. The fully reversible field-induced magneto-structural transition is typically accompanied by the presence of large hysteresis losses. In the presence of hysteresis but especially for large hysteresis, the magnetization, $\mathrm{M}$, at a given temperature is not a single-valued function of the magnetic field, $\mathrm{H}$, but it depends on the magnetic history. This observation raises the very important issue about the applicability of using the Maxwell relation for computing the $\Delta \mathrm{S}$ peak from magnetic data when large hysteresis losses are present.

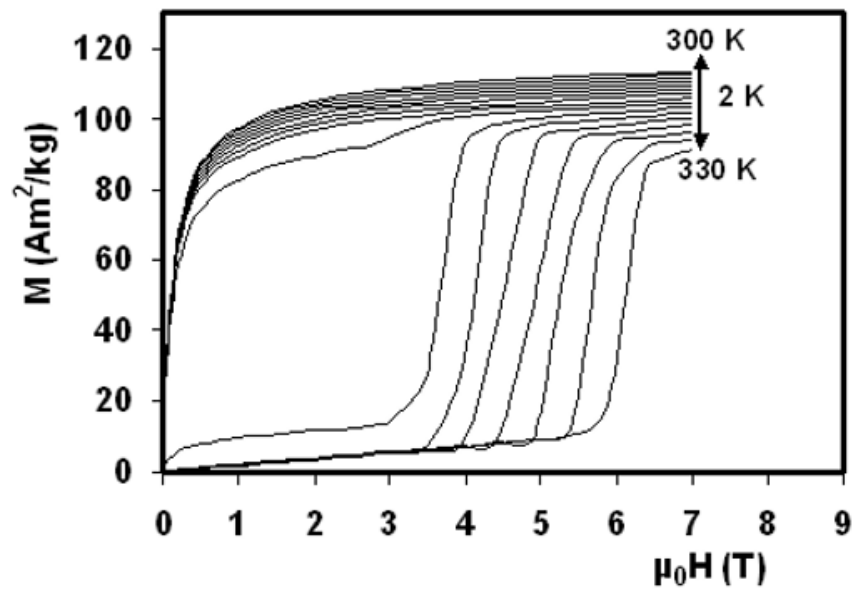

Fig. 1. Isothermal magnetization curves of MnAs close to the Curie temperature $\left(T_{C}=317 \mathrm{~K}\right)$

Figure 1 shows the isothermal magnetization traces recorded near Curie temperature for a sample of MnAs. These traces which show a metamagnetic transition close to $\mathrm{T}_{\mathrm{C}}$ reveal a mixture of two magnetic phases (ferromagnetic and paramagnetic) around the transition region as a consequence of the hysteresis phenomenon. Below the Curie temperature $\left(T_{C}=317 \mathrm{~K}\right)$, the magnetization curves are characteristic of ferromagnetic systems and metamagnetism is observed above this temperature. As shown in Figure 1, the traces exhibit a magnetization jump $\Delta \mathrm{M}$ at the metamagnetic transition over a field range $\Delta \mathrm{H}_{\mathrm{C}}$, where $\mathrm{H}_{\mathrm{C}}$ is the critical field defined at the inflexion point in the transition region. Using $\mathrm{MR}, \Delta \mathrm{S}$ is related to the bulk magnetization, the temperature and the magnetic field:

$$
\Delta S(T, \Delta H)=\int_{0}^{H \max }\left(\frac{\partial M}{\partial T}\right)_{H^{\prime}} d H^{\prime}
$$

From isothermal magnetization curves, $\Delta \mathrm{S}$ can be calculated numerically by :

$$
\Delta S=\sum_{i} \frac{M_{i+1}-M_{i}}{T_{i+1}-T_{i}} \Delta H_{i}
$$

where $M_{i+1}$ and $M_{i}$ are the magnetization values measured in a given field $H$, at temperatures $T_{i+1}$ and $T_{i}$, respectively. The calculated $\Delta \mathrm{S}$ is then equivalent to the area between two successive magnetic isotherms normalized by the corresponding temperature. Based on equation 2 and figure 1 data, the entropy change as a function of temperature and magnetic field of the here studied MnAs is given in figure 2 (squares). $-\Delta \mathrm{S}$ presents a maximum value of about $130 \mathrm{~J} / \mathrm{kg} \mathrm{K}$ at $\mathrm{T}_{\mathrm{C}}=317 \mathrm{~K}$, which is more than 4 times higher than that reported by Wada et al [3]. However, this obtained "colossal" value determined using equation 2 is spurious and not correct. This is attributed to the inadequate use of the Maxwell relation when two magnetic phases coexist in the materials which is the case of the here studied MnAs. In FOMT materials (ferromagnets) with phase-separated state, 
only the parts of volume with paramagnetic phase contribute to the magnetocaloric effect when the metamagnetism is induced to reach a complete ferromagnetic phase. Using directly MR, large parts of the normalized area between two successive isotherms near $T_{C}$ is included in the calculation of the entropy change because of the remaining volume portions of the ferromagnetic phase. The latter does not contribute to the MCE, but its presence distorts the integration process leading to wrong and overestimated values of $\Delta \mathrm{S}$. This latter can be evaluated accurately by using Clausius-Clapeyron equation, in particular when the ferromagnetic state is completely saturated after the metamagnetic transition as shown for example in figure 1. In $\mathrm{C}-\mathrm{C}$ equation, $\Delta \mathrm{S}$ is directly related to the magnetization jump induced by the field for a given temperature, it is given by:

$$
\Delta S=-\Delta M \frac{d H_{C}}{d T}=-\Delta M\left(\frac{d T_{C}}{d H}\right)^{-1}
$$

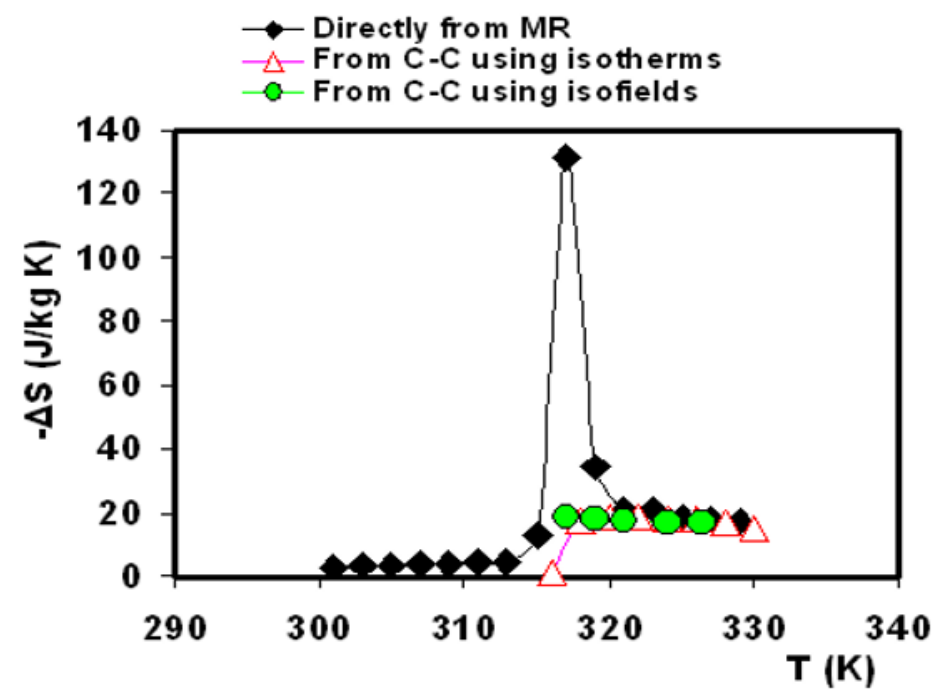

Fig. 2. Isothermal entropy change for MnAs calculated directly from MR under $7 \mathrm{~T}$ (squares), From $\mathrm{C}-\mathrm{C}$ equation using isotherms (triangles) and isofields (circles).

$\Delta \mathrm{M}$ can be determined as the difference of magnetization between the linear extrapolation of magnetization isotherms below and above the critical field $\mathrm{H}_{\mathrm{C}}$ which can be obtained from data of figure 1. For this doing, $\mathrm{dH}_{\mathrm{C}} / \mathrm{dT}$ is estimated to be about $0.2 \mathrm{~T} / \mathrm{K}$. The corrected $\Delta \mathrm{S}$ is then given in figure 2 (triangles). The maximum obtained $\Delta \mathrm{S}$ using this approach is about $20 \mathrm{~J} / \mathrm{kg} \mathrm{K}$ instead 130 $\mathrm{J} / \mathrm{kg} \mathrm{K}$ deduced directly from MR which is more realistic and comparable with that reported in Ref.3. $\Delta \mathrm{S}$ can also calculated using $\mathrm{C}-\mathrm{C}$ equation and the thermomagnetic measurements (isofields) given in figure 3. In this case $\mathrm{dT}_{\mathrm{C}} / \mathrm{dH}$ should be determined, it is estimated to be about $5 \mathrm{~K} / \mathrm{T}$ and $\Delta \mathrm{M}$ correspond to the magnetization jump at $\mathrm{T}_{\mathrm{C}}$. The obtained result (circles) is similar to that determined from isothermal curves. However, as demonstrated by Liu et al [15], realistic values of the entropy change can be also obtained from Maxwell relation when this latter is integrated only on the metamagnetic region.

In recent years, spurious and overestimated values of the magnetic entropy change were also reported frequently in the literature for the Ni-Mn-In Heusler alloy based materials [18]. Generally, these materials where the magnetism is basically governed by Mn magnetic moment, undergo two magnetic transition. A classical second order transition from paramagnetic to ferromagnetic state takes place at $T_{C}$ and a first order martensitic transformation at $T_{m}$ below $T_{C}$. The martensitic transition induced by field/or temperature is accompanied by a magnetic phase transition from the antiferromagnetic (AF) to the ferromagnetic state. Near $\mathrm{T}_{\mathrm{m}}$, a large hysteresis is generally observed in these materials which explain the presence of the mixed magnetic state constituted of $\mathrm{AF}$ and 
Ferro phases. In this case, only the antiferromagnetic phase contribute to the magnetocaloric effect when is changed by the magnetic field to the ferromagnetic state. The presence of the ferromagnetic phase distorts the integration process leading to colossal and non realistic value of the entropy change, which explain for example the data reported in reference [19]. The spurious $\Delta S$ values reported in the literature for some Heusler alloys were confirmed more recently by adiabatic temperature change measurements performed by Khovaylo et al [20]. Taking the $\mathrm{Ni}_{2.19} \mathrm{Mn}_{0.81} \mathrm{Ga}$ compound as a case study, they found that $\Delta \mathrm{T}_{\mathrm{ad}}(\sim 1 \mathrm{~K})$ measured in the vicinity of a first-order magneto-structural transition is of order of magnitude smaller than that expected from magnetization measurements and specific heat data. More details can be found in Ref.20.

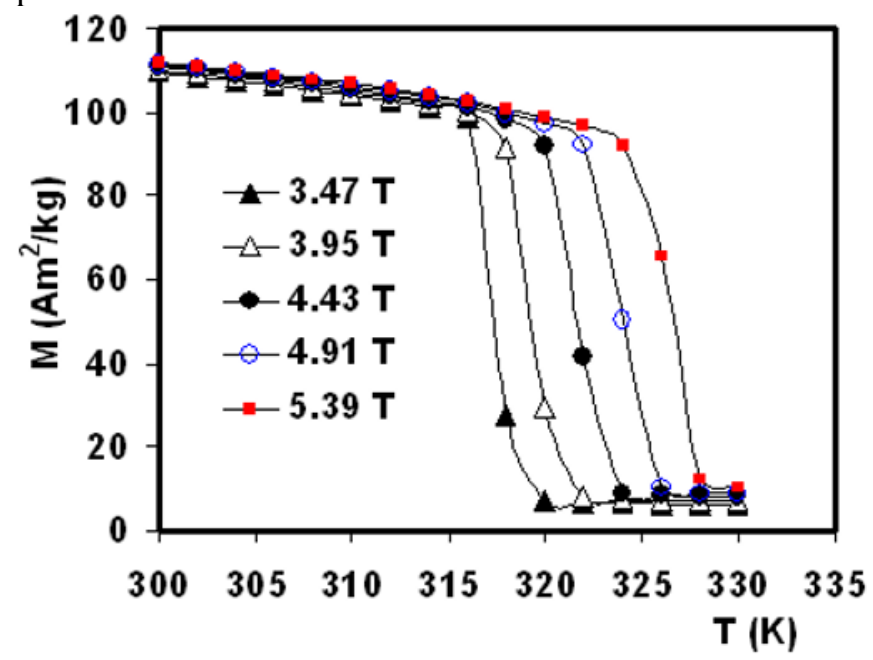

Fig. 3. Thermomagnetic curves (isofields) for different magnetic fields.

More recently, it was shown that the magnetic entropy change of materials (ferromagnets) with hysteretic first-order transitions can be calculated via the Maxwell relation [21]. The reported method suggest that a loop process may approach a reasonable $\Delta \mathrm{S}$, which means one should firstly heat sample to paramagnetic state in zero field before performing each isotherms to avoid the hysteresis effect and residual ferromagnetic phase from the last temperature magnetic loop. This method was applied to some selected materials and the calculated values are much lower compared with those deduced directly without loop process. However, the obtained $\Delta S$ using the method described in Ref. 21 remain much larger compared with the best magnetocaloric materials. This is probably explained by the non-respect of the equilibrium state necessary for the application of the MR. In addition, another source of errors, arises from the imperfect temperature control when performing the loop process and/or the isotherms measurement in hysteretic FOMT materials which results in an incorrect normalization of the areas enclosed between adjacent magnetization [22]. In order to validate the method reported in Ref. 21, and to see the difference between the real values of $\Delta \mathrm{S}$ and those estimated, specific heat measurement should be performed in equilibrium conditions.

\section{First order materials with non-phase-separated state}

Many FOMT materials present a non-phase-separated sate close to the phase transition like $\mathrm{Gd}_{5} \mathrm{Ge}_{2} \mathrm{Si}_{2}$ [2]. Taking this material as a case of study, Giguère et al. [10] have shown that the entropy change calculated from MR was overestimated compared to that obtained from the C-C equation. They concluded that MR was not usable for materials with first order magnetic transition such as $\mathrm{Gd}_{5} \mathrm{Si}_{2} \mathrm{Ge}_{2}$. According to Giguère et al work [10], the C-C equation is more appropriated for this kind of materials. However, the low value of $\Delta \mathrm{S}$ reported for $\mathrm{Gd}_{5} \mathrm{Ge}_{2} \mathrm{Si}_{2}$ in $\mathrm{Ref} .10$ is due to the fact that $\mathrm{C}-\mathrm{C}$ equation evaluates the $\mathrm{MCE}$ only related to the magnetization jump at the metamagnetic transition. The entropy change related to the magnetization evolution before and after 
the transition was not taken into account. This discrepancy can be shown here for the compound $\mathrm{HoCo}_{2}$ which presents similar magnetic behaviour as $\mathrm{Gd}_{5} \mathrm{Ge}_{2} \mathrm{Si}_{2}$.

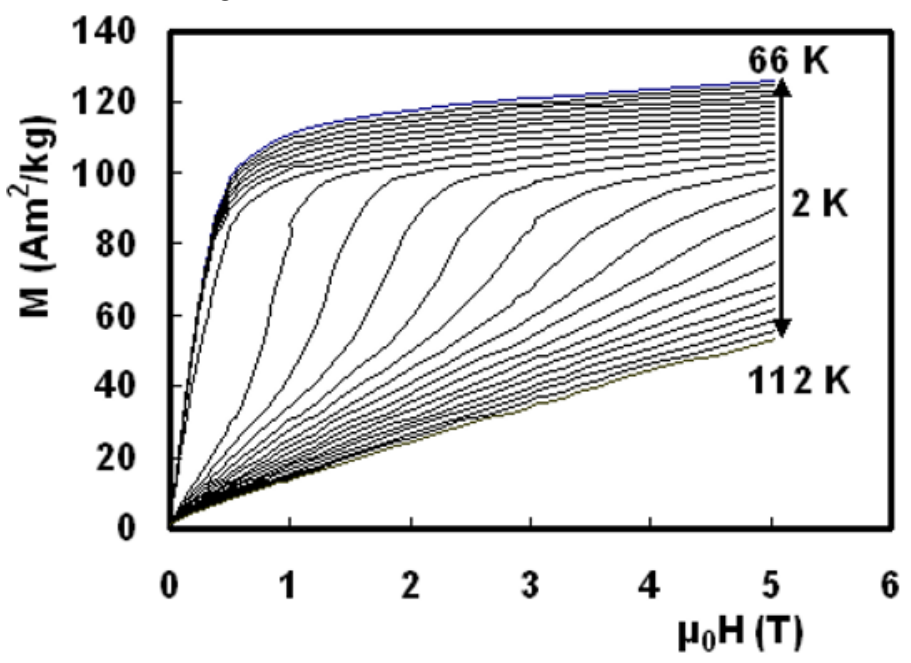

Fig. 4. Isothermal magnetization curves of $\mathrm{HoCo}_{2}$ close to the Curie temperature $\left(\mathrm{T}_{\mathrm{C}}=81 \mathrm{~K}\right)$.

According to magnetic measurements, the Itinerant-Electron-Metamagnetic-Transition (IEMT) associated with the first order character of the transition at the Curie temperature $T_{C}$ is clearly observed in $\mathrm{HoCO}_{2}$. The IEMT is a field induced transition due to the change in the band structure of $3 \mathrm{~d}$ electrons by applying a magnetic field. No double phase is observed for $\mathrm{HoCo}_{2}$ as shown in figure 4. Figure 5 shows the magnetic entropy change as a function of temperature given for $5 \mathrm{~T}$ and determined by integrating directly $\mathrm{MR}$ (circles). $\Delta \mathrm{S}$ deduced from $\mathrm{C}-\mathrm{C}$ equation is also given for comparison (triangles). For $\mathrm{HoCO}_{2}$, the maximum values of $\Delta \mathrm{S}$ calculated with $\mathrm{MR}$ for a magnetic field change from 0 to $5 \mathrm{~T}$ at $\mathrm{T}_{\mathrm{C}}$ is found to be about $18 \mathrm{~J} / \mathrm{kg} \mathrm{K}$ instead $15 \mathrm{~J} / \mathrm{kg} \mathrm{k}$ when using C-C equation. The $\mathrm{C}-\mathrm{C}$ resulting traces scale well with those of $\mathrm{MR}$, but $\Delta \mathrm{S}$ can be higher or lower depending on the maximum value of the applied magnetic field, being clearly below MR values if e.g. $\mathrm{H}_{\max } \gg \mathrm{H}_{\mathrm{C}}$ (Fig.5). This discrepancy between MR and C-C results in this case, disappears when one calculates the entropy change in the transition range using the Maxwell relation defined as:

$$
\Delta S\left(T, \Delta H_{C}\right)=\int_{H_{C}-\frac{\Delta H_{C}}{2}}^{H_{C}+\frac{\Delta H_{C}}{2}}\left(\frac{\partial M}{\partial T}\right) d H^{\prime}
$$

The newly obtained results are plotted in Figures 5 (solid line), showing that the $\Delta S\left(T, \Delta H_{C}\right)$ values are in good agreement with those of the C-C based analysis. This suggests that the calculation of the entropy changes using the Maxwell relation evaluated within transition region and using the Clausius-Clapeyron equation, are equivalent for the $1^{\text {st }}$ order magnetic transition provided that the MR is applied within the $\Delta \mathrm{H}_{\mathrm{C}}$ field range but not from zero to a given field. Besides, for a complete entropy change, MR must be integrated on the full magnetic field range and $\mathrm{C}-\mathrm{C}$ values should be completed by taking into account the region outside phase transition. 


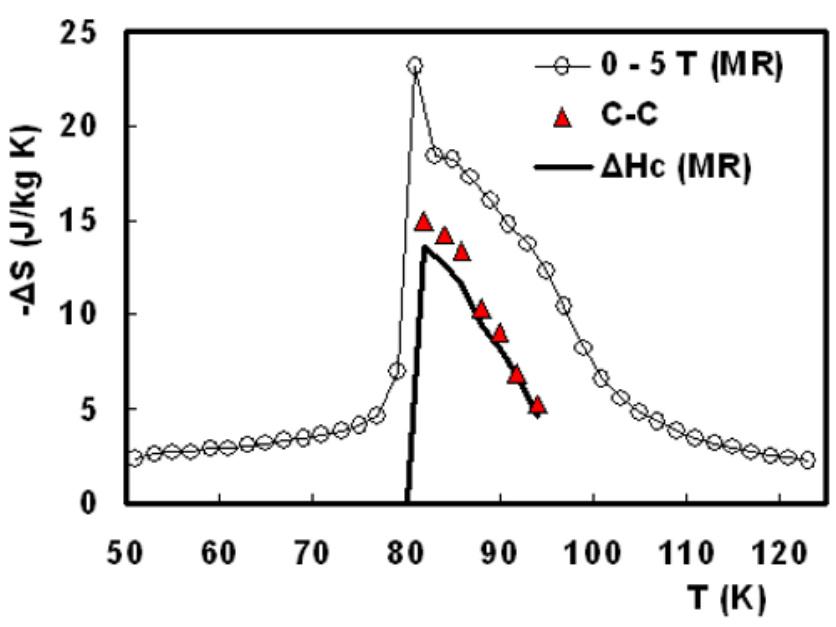

Fig. 5. Isothermal entropy change for $\mathrm{HoCO}_{2}$ calculated directly from MR under $5 \mathrm{~T}$ (circles), From $\mathrm{C}-\mathrm{C}$ equation using isotherms (triangles) and from MR integrated only on the transition region (solid line).

\section{Effect of the demagnetization field on the MCE}

Generally in the literature, the most reported data for the magnetocaloric effect are not corrected for demagnetization, and the published results are associated directly to the external field. However, when subjecting a magnetic material to an external magnetic field, the resulting internal magnetic field depends on the sample shape and it is different from the applied field [23]. Accordingly, the measured magnetocaloric effect is a result of the internal field (local field) and not the consequence of the external field. The internal magnetic field is the effective field $\left(\mathrm{H}_{\mathrm{Eff}}\right)$ acting on the material physical parameters such as magnetization and specific heat, defining the magnitude of its magnetocaloric effect. Under the effect of an external magnetic field, the tested sample creates in the opposite direction the so-called demagnetization field cancelling out a part of the applied field, resulting in spurious values of the measured MCE. It is then extremely important to always report magnetocaloric properties as a function of the internal magnetic field. Besides, it should be noted that the demagnetization field in non-ellipsoidal bodies is not uniform [24]. It is a function of the space in the material. Therefore, in order to define a constant demagnetizing factor, it was necessary to define some sort of averaging of this field. Aharoni [24] has proposed a simple analytical expression for the demagnetization factors $\left(\mathrm{N}_{\mathrm{d}}\right)$ of the general rectangular prism. For other geometries, $\mathrm{N}_{\mathrm{d}}$ can be also determined using simple approaches [25]. The average internal field can be obtained by subtracting the demagnetization field $H_{d}=-N_{d} M$ from the external field $H_{0}$,

$$
H_{\text {eff }}=H_{0}-N_{d} M
$$

$M$ is the magnetization. The direct evaluation of the magnetocaloric effect exhibited by a sample of gadolinium with the dimensions $2 * 2 * 2 \mathrm{~mm}^{3}$, is shown in figure 6 for an external field of $1 \mathrm{~T}$. 


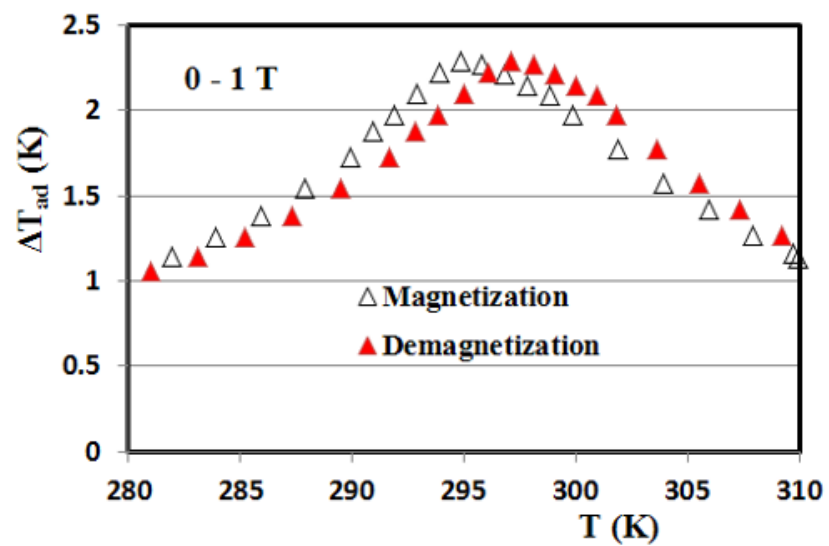

Fig. 6. Measured adiabatic temperature change on a sample of $\mathrm{Gd}\left(2 * 2 * 2 \mathrm{~mm}^{3}\right)$ during magnetization and demagnetisation, as a function of temperature under an external field of $1 \mathrm{~T}$.

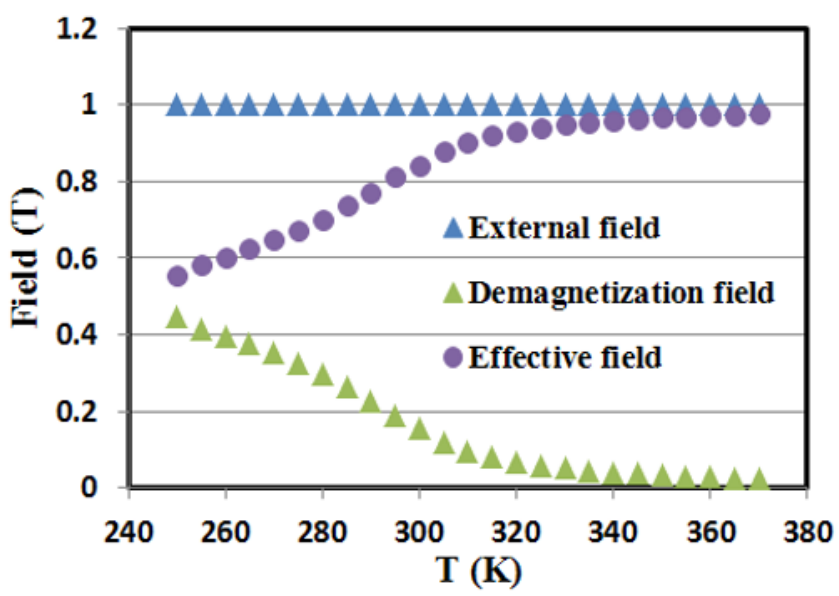

Fig. 7. Effect of the demagnetization field on the effective magnetic field.

The measurements were performed in adiabatic conditions using the facilities of Néel Institute, Grenoble. Both curves represent, $\Delta \mathrm{T}_{\mathrm{ad}}$ exhibited by $\mathrm{Gd}$ upon application and removal of the magnetic field. The maximum of the data recorded during field removal is shifted to a high temperature. This is attributed to the reversibility of the MCE. The distance between the maximums is equivalent to the maximum adiabatic temperature change. From data of figure 6 , under an external field of $1 \mathrm{~T}$, the $\Delta \mathrm{T}_{\mathrm{ad}}$ peak at $\mathrm{T}_{\mathrm{C}}=294 \mathrm{~K}$ is $2.3 \mathrm{~K}$, which is lower compared to the standard value of the Gd MCE $(3 \mathrm{~K} / \mathrm{T})$. This is attributed as explained before to the demagnetization effect. Using equation (5) and the magnetization measurements, we present in figure 7 the temperature dependence of the effective magnetic field inside the Gd sample $\left(2 * 2 * 2 \mathrm{~mm}^{3}\right)$. The demagnetization factor of this latter is found to be 0.33 . The evolution of the demagnetization field as a function of temperature is also given. As shown in figure 7, a large deviation is observed at low temperatures between the external and the effective (internal) field due to the large value of the magnetization in this temperature range corresponding to the ferromagnetic phase. With the increasing of temperature, the effective field increases approaching the external field in the paramagnetic state (high temperatures). Around $\mathrm{T}_{\mathrm{C}}=294 \mathrm{~K} \mathrm{H}_{\mathrm{Eff}}$ is about $0.8 \mathrm{~T}$. This is different from the applied external field which is equal to $1 \mathrm{~T}$. Consequently, the correction for the effect of demagnetization is of great importance for accurate measurement of the MCE. The normalized adiabatic temperature change (during magnetization), respect to the effective field is plotted in figure 8 . Therefore, taking into 
account the demagnetization effect, and after correcting the data of figure 6 , the new value (intrinsic value) of the $\Delta \mathrm{T}_{\mathrm{ad}}$ peak for $\mathrm{Gd}$ becomes $2.8 \mathrm{~K}$ for $1 \mathrm{~T}$ instead $2.3 \mathrm{~K}$.

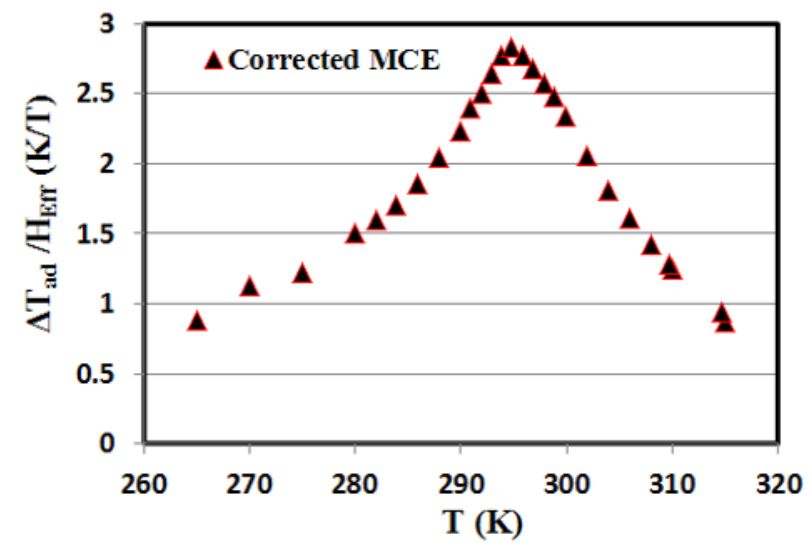

Fig. 8. Corrected MCE taking into account the demagnetization effect.

\section{Conclusions}

In conclusion, the Maxwell relation can be used to estimate the entropy change displayed by FOMT magnetic compounds when these latter present a non-phase-separated state. The observed difference usually reported between application of the Maxwell relation and the Clausius-Clapeyron equation in these materials can be attributed to the fact that the (C-C) equation does not account for the induced entropy change below and above transition region where metamagnetism takes place. However, for FOMT materials with mixed phases, MR gives spurious values of $\Delta \mathrm{S}$. More realistic values of $\Delta \mathrm{S}$ can be obtained by using $\mathrm{C}$-C equation, particularly when the ferromagnetic phase is completely saturated. The influence of the demagnetization effect on the accurate measurement of the magnetocaloric effect was also investigated. The calculation of the effective magnetic field inside a sample of Gd demonstrates that a large part of the applied external field can be cancelled due to the demagnetization field, reducing consequently the magnetocaloric effect. Therefore, the measured $\Delta \mathrm{T}_{\mathrm{ad}}$ as well as the entropy change and specific heat should be corrected taking into account the demagnetization effect. This allows a good estimation for the magnetocaloric properties.

\section{Acknowledgments}

We are grateful to INTERREG IV-A France-Suisse for financing this work.

\section{References}

1. E. Warburg, Ann. Phys. Chem. 13141 (1881).

2. V. K. Pecharsky, and K.A. Gschneidner., Jr. Phys. Rev. Lett. 78, 4494 (1997).

3 H. Wada and Y. Tanabe, Appl. Phys. Lett. 79, 3302 (2001).

4. O. Tegus, E. Brück, K.H.J. Buschow and F.R. de Boer, Nature. 415, 150 (2002).

5. Y.F. Chen, F. Wang, B.G. Shen, F.X. Hu, Z.H. Cheng, G.J. Wang and J.R. Sun, Chin. Phys. Soc. Ltd.7, 74 (2002)

6. A. Fujita, S. Fujieda, Y. Hasegawa and K. Fukamichi, Phys. Rev. B. 67, 104416 (2003).

7. F. X. Hu, B.G. Shen, J.R. Sun, G.J. Wang and Z.H. Cheng, Appl. Phys. Lett. 80, 826 (2002).

8. M. Balli, D. Fruchart and D. Gignoux, J. Phys.: Condens. Matter. 19, 236230 (2007).

9. M. Balli, D. Fruchart and D. Gignoux, Appl. Phys. Lett. 92, 232505 (2008). 
10. A. Giguère, M. Földeaki, B. Ravi Gopal, R. Chahine, T. K. Bose, A. Frydman, and J. A. Barclay, Phys. Rev. Lett. 83, 2262 (1999).

11. K. A. Gschneidner, Jr., V. K. Pecharsky, E. Brück, H. G. M. Duijn and E. M. Levin, Phys. Rev. Lett. 85, 4190 (2000).

12. J. R. Sun, F. X. Hu, and B. G. Shen, Phys. Rev. Lett. 85, 4191 (2000)

13. M. Földeaki, R. Chahine, T. K. Bose, A. Frydman, and J. A. Barclay, Phys. Rev. Lett. 85, 4191 (2000).

14. M. Balli, D. Fruchart, D. Gignoux and R. Zach, Appl. Phys. Lett. 95, 072509 (2009).

15. G. J. Liu, J. R. Sun, J. Shen, B. Gao, H. W. Zhang, F. X. Hu and B. G. Shen, Appl. Phys. Lett. 90, 032507 (2007)

16. J. S. Amaral, V.S. Amaral, J. Magn. Magn. Mater. 322, 1552 (2010).

17. F. Casanova, X. Batlle, A. Labarta, J. Marcos, L. Manosa and A. Planes, Phys.Rev.B. 100401(R) (2002).

18. A. Planes, L. Manosa and M. Acet, J. Phys.: Condens. Matter. 21, 233201 (2009).

19. A. K. Pathak, I. Dubeko, S. Stadler and N. Ali, J. Phys.D: Appl Phys. 41, 202004 (2008).

20. V. V. Khovaylo, K. P. Skokov, Y. S. Koshkid'ko, V. V. Koledov, V. G. Shavrov, V. Buchelnikov, S. V. Taskaev, H. Miki, T. Takagi and A. N. Vasiliev, Phys.Rev.B. 78, 060483 (2008)

21. L. Caron, Z. Q. Ou, T. T. Nguyen, D. T. CamThanh, O. Tegus, E. Bruck, J. Magn. Magn. Mater 321, 3559 (2009).

22. V. K. Pecharsky, K. A. Gschneidner Jr., Ya. Mudryk, Durga Paudyal, J. Magn. Magn. Mater 321, 3541 (2009).

23. C. R. H. Bahl and K. K. Nelson, J. App. Phys. 105, 013916 (2009).

24. A. Aharoni, J. Appl. Phys. 83, 3432 (1998).

25. Magnétisme, Fondement, Ed: E. du Trémolet de Lacheisserie, EDP Sciences, 2000. 